\title{
Mutant alleles associated with late-onset ornithine transcarbamylase deficiency in male patients have recurrently arisen and have been retained in some populations
}

\author{
Sanae Numata ${ }^{1}$, Yoshiro Koda ${ }^{2}$, Kenji Ihara ${ }^{3}$, Tomo Sawada ${ }^{4}$, Yoshiyuki Okano ${ }^{4}$, Toshinobu Matsuura ${ }^{5}$, \\ Fumio Endo ${ }^{5}$, Han-Wook Yoo ${ }^{6}$, Jose A Arranz ${ }^{7}$, Vicente Rubio ${ }^{8}$, Bendicht Wermuth ${ }^{9}$, Nicholas Ah Mew ${ }^{10}$, \\ Mendel Tuchman ${ }^{10}$, Jason R Pinner ${ }^{11}$, Edwin P Kirk ${ }^{11}$ and Makoto Yoshino ${ }^{1}$
}

We performed haplotype analysis using nine single nucleotide polymorphisms in the ornithine transcarbamylase gene to explore the ancestral origins of three mutations associated with late-onset phenotype in male patients: p.R40H, p.R277W and p.Y55D. Overall, 8 haplotypes were defined among 14 families carrying p.R4OH, 5 families carrying p.R277W and 2 families with p.Y55D mutations. Of nine Japanese families carrying p.R40H, eight exhibited haplotype (HT)1, whereas the other family harbored HT2. Among three Caucasian families, one Spanish and one Australian family bore HT3; one Austrian family had HT4. Two US patients harbored HT2 and HT4. Among families carrying p.R277W, HT5 was found in one Japanese, one Korean and one US family. Two other US families had HT2 and HT6. Two families carrying p.Y55D, both Japanese, shared HT1. These results indicate that the p.R4OH mutation has arisen recurrently in all populations studied, although there is evidence for a founder effect in Japan, with most cases probably sharing a common origin, and to a lesser extent in subjects of European ancestry (HT3). It is evident that p.R277W mutation has recurred in discrete populations. The p.Y55D mutation appears to have arisen from a common ancestor, because this transversion (c.163T $>$ G) occurs rarely.

Journal of Human Genetics (2010) 55, 18-22; doi:10.1038/jhg.2009.113; published online 6 November 2009

Keywords: haplotype; late-onset; male; ornithine transcarbamylase deficiency; recurrent mutation; single nucleotide polymorphism

\section{INTRODUCTION}

Ornithine transcarbamylase (OTC) deficiency (OMIM no. 311250) is the most common inherited disorder of the urea cycle and is transmitted as an X-linked trait.

The locus of the gene encoding OTC is on the short arm of the $\mathrm{X}$ chromosome within band $\mathrm{Xp} 21.1 .^{1}$ The gene spans $74 \mathrm{~kb}$ with an open reading frame of 1062 nucleotides distributed into 10 exons and 9 introns. ${ }^{2,3}$ The phenotypes of females heterozygous for a mutant OTC allele vary from asymptomatic carrier state to overt, even fatal disease, depending first on the nature of the gene mutation, second on X-inactivation pattern and third on other genes and environmental factors. In contrast, in hemizygous male patients, the phenotype is determined by the nature of mutation and other yet unknown factors (other genes/environment). Such male patients most commonly develop symptoms of hyperammonemia in the neonatal period or in early infancy and their disease is often fatal. ${ }^{4}$ However, there are some male patients in whom the onset of the disease is delayed until the preschool age period ${ }^{5}$ through to adulthood. ${ }^{6,7}$ Some affected males within the same families may remain asymptomatic for life. ${ }^{8}$ Their condition is now recognized as 'late-onset OTC deficiency in male patients', accounting for $\sim 30 \%$ of male patients. ${ }^{9}$ Such male patients reproduce at a fitness value of $0.49 .{ }^{10}$ Although the majority of mutations at human OTC locus are 'private', being observed in single families only, ${ }^{11}$ several mutations have been observed repeatedly in discrete families, ${ }^{12-14}$ mainly affecting CpG dinucleotides. Among those, the c.119G $>$ A (p.R40H) and c.829C $>$ T (p.R277W) mutations have been repeatedly reported in multiple ethnicities. In our previous series of Japanese families, the c.119G $>$ A (p.R40H) mutation was encountered in a cluster. ${ }^{7,10,15}$ In addition, we identified another novel mutation, c.163T $>$ G (p.Y55D) in two discrete families. ${ }^{10,16}$ It is not

${ }^{1}$ Department of Pediatrics and Child Health, Kurume University School of Medicine, Kurume, Japan; ${ }^{2}$ Department of Forensic Medicine and Human Genetics, Kurume University School of Medicine, Kurume, Japan; ${ }^{3}$ Department of Pediatrics, Graduate School of Medicine, Kyushu University, Fukuoka, Japan; ${ }^{4}$ Department of Pediatrics, Osaka City University Graduate School of Medicine, Osaka, Japan; ${ }^{5}$ Department of Pediatrics, Graduate School of Medicine, Kumamoto University, Kumamoto, Japan; ${ }^{6}$ Department of Pediatrics, Asan Medical Center, Seoul, Korea; 7 Unitat de Metabolopaties, Hospital Universitari Materno-Infantil Vall d'Hebron, Passeig de la Vall d'Hebron, Barcelona, Spain; ${ }^{8}$ Instituto de Biomedicina de Valencia (IBV-CSIC) and CIBERER-ISCIII, Valencia, Spain; 9Universitätsinstitut für Klinische Chemie/Molekulare Diagnostik, Inselspital, Bern, Switzerland; ${ }^{10}$ Children's National Medical Center, Washington DC, USA and ${ }^{11}$ Department of Medical Genetics, Sydney Children's Hospital, Randwick, Australia

Correspondence: Professor M Yoshino, Department of Pediatrics and Child Health, Kurume University School of Medicine, 67, Asahi-machi, Kurume, Fukuoka 830-0011, Japan. E-mail: yoshino@med.kurume-u.ac.jp

Received 3 August 2009; revised 2 October 2009; accepted 5 October 2009; published online 6 November 2009 
known whether or not these mutations share a common ancestral origin or have arisen recurrently.

Polymorphic sites in the human OTC gene having potential for family tracking were reported previously. These include single nucleotide substitutions, insertions and short tandem repeats. ${ }^{9,17-22}$ After completion of the HapMap Project, single nucleotide polymorphisms (SNPs) have become available for haplotype analysis on a given gene.

However, SNP-based haplotype analysis of the human OTC locus has not been reported previously. We aimed to determine the haplotypes of these mutant OTC alleles to explore the origins of these mutations.

\section{MATERIALS AND METHODS}

Families

A total of 14 families with the c.119G $>$ A (p.R40H) mutation, 5 families with the c.829C $>$ T (p.R277W) mutation and 2 families with the c. $163 \mathrm{~T}>\mathrm{G}$ (p.Y55D) mutation were studied. The 14 families with the p.R40H mutation consisted of 9 families from Japan, 1 family each from Spain, Australia and Austria, and 2 families from the United States. The families with p.R277W included 1 family from Japan, 1 family from Korea and 3 families from US. Two families with p.Y55D were both Japanese. The probands were all male, except the proband in family 11, a 13-year-old symptomatic girl who carried the p.R40H mutation, as did her asymptomatic mother. Families 1-9, 15, 20 and 21 were Japanese; family 16, Korean; and families 10-12, Caucasians.

None of the families in the present series were known to be related to each other. Demographic information, including ethnic background, of the US families $(13,14,17-19)$ was not available because of the US Personal Data Protection Act. Haplotype analysis was performed on the proband from each family and their relatives, when specimens were available from them. In family 11, the DNA specimens from parents of the proband were also analyzed to determine the mutation-bearing allele. In family 12 , the DNA specimen of the proband was prepared from the liver tissue obtained after it had been inadvertently transplanted to a woman. ${ }^{23}$

\section{Haplotype analysis}

A total of 9 tagged SNPs were selected on the Haploview ${ }^{24}$ with $r^{2}$ of 0.80 and minor-allele frequency of 0.05 (Table 1). The haplotype frequencies of Japanese in Tokyo (JPT), Utah residents with Northern and Western European ancestry from CEPH collection (CEU) and Yoruban in Ibadan, Nigeria (YRI) were available on http://hapmap.ncbi.nlm.nih.gov/index.html.en. The nucleotide combinations in Pan troglodytes (chimpanzee) were obtained on http://www.ensembl. org/index.html to estimate the human ancestral alleles. The nucleotide in the polymorphic site 6 (rs5963421 for human) for P. troglodytes was represented by that in Pongo pygmaeus abelii (orangutan), because this nucleotide was not available for $P$. troglodytes.

The SNPs were determined by the TaqMan probe-based real-time PCR on LightCycler LC-480 (Roche Diagnostics GmbH, Mannheim, Germany). The PCR was carried out in a total volume of $20 \mu$, containing $20 \mathrm{ng}$ genomic DNA, $10 \mu \mathrm{l}$ LC-480 Probe Master (Roche) and $1 \mu \mathrm{l} 20 \times$ Probe/Primer Mix (Applied Biosystems, LLC, Foster City, CA, USA). The probes used were labeled as either FAM or VIC. The temperature was programmed as follows: a pre-incubation step at $95^{\circ} \mathrm{C}$ for $10 \mathrm{~min}$, followed by 40 cycles of denaturation at $95^{\circ} \mathrm{C}$ for $10 \mathrm{~s}$, annealing at $60^{\circ} \mathrm{C}$ for $60 \mathrm{~s}$ and extension at $72^{\circ} \mathrm{C}$ for $1 \mathrm{~s}$. At the end of the annealing step, the fluorescence signal was measured. After the PCR reaction, the temperature was decreased to $40^{\circ} \mathrm{C}$ as a cooling step.

\section{Ethical considerations}

The Ethical Committee of Kurume University approved this project and specimens were obtained in accordance with respective institutional bioethical standards and relevant bioethical regulations or guidelines in each country.

\section{RESULTS}

Haplotypes defined in the families and their allelic frequency and heterozygosity

The data on the nine SNPs employed and the haplotypes determined in the families by the use of these SNPs are summarized in Table 1. Six discrete haplotypes (HT's) (1-6) were found among the probands. The parents of the female proband in family 11 carried two additional HT's, 7 and 8.

Table 1 Haplotypes generated by single-nucleotide polymorphism in human ornithine transcarbamylase locus

\begin{tabular}{|c|c|c|c|c|c|c|c|c|c|c|c|c|c|c|}
\hline \multirow[b]{2}{*}{ Polymorphic sites } & \multirow[b]{2}{*}{ Tagged SNPS } & \multirow[b]{2}{*}{ Nucleotide } & \multicolumn{3}{|c|}{ Allele frequency heterozygosity } & \multicolumn{9}{|c|}{ Haplotype } \\
\hline & & & $J P N$ & CEU & $Y R I$ & 1 & 2 & 3 & 4 & 5 & 6 & 7 & 8 & Pan troglodytes \\
\hline 1 & rs5917576 & $\mathrm{G} / \mathrm{A}$ & $\begin{array}{l}0.91 / 0.09 \\
0.164\end{array}$ & $\begin{array}{l}0.50 / 0.50 \\
0.500\end{array}$ & $\begin{array}{l}0.83 / 0.17 \\
0.282\end{array}$ & G & G & G & A & $\mathrm{G}$ & G & A & G & G \\
\hline 2 & rs17274134 & $\mathrm{G} / \mathrm{C}$ & $\begin{array}{l}0.53 / 0.47 \\
0.498\end{array}$ & $\begin{array}{l}0.08 / 0.92 \\
0.147\end{array}$ & $\begin{array}{l}0.19 / 0.81 \\
0.308\end{array}$ & G & C & C & C & C & C & C & C & C \\
\hline 3 & rs6417794 & $\mathrm{G} / \mathrm{C}$ & $\begin{array}{l}0.77 / 0.23 \\
0.354\end{array}$ & $\begin{array}{l}0.79 / 0.21 \\
0.332\end{array}$ & $\begin{array}{l}0.88 / 0.12 \\
0.211\end{array}$ & $\mathrm{G}$ & C & C & G & G & C & G & G & G \\
\hline 4 & rs6609709 & $\mathrm{G} / \mathrm{A}$ & $\begin{array}{l}0.11 / 0.89 \\
0.196\end{array}$ & $\begin{array}{l}0.23 / 0.77 \\
0.354\end{array}$ & $\begin{array}{l}0.30 / 0.70 \\
0.420\end{array}$ & A & A & A & A & G & A & A & $A$ & A \\
\hline 5 & rs2235125 & $\mathrm{G} / \mathrm{A}$ & $\begin{array}{l}0.38 / 0.62 \\
0.471\end{array}$ & $\begin{array}{l}0.63 / 0.36 \\
0.454\end{array}$ & $\begin{array}{l}0.22 / 0.78 \\
0.343\end{array}$ & A & G & G & G & A & G & A & $A$ & $A$ \\
\hline 6 & rs5963421 & $\mathrm{A} / \mathrm{T}$ & $\begin{array}{l}0.82 / 0.18 \\
0.295\end{array}$ & $\begin{array}{l}0.60 / 0.40 \\
0.480\end{array}$ & $\begin{array}{l}0.92 / 0.08 \\
0.147\end{array}$ & A & $A$ & $\mathrm{~T}$ & $\mathrm{~T}$ & A & A & A & $A$ & $A$ \\
\hline 7 & rs17274141 & $\mathrm{C} / \mathrm{T}$ & $\begin{array}{l}0.46 / 0.53 \\
0.488\end{array}$ & $\begin{array}{l}0.13 / 0.87 \\
0.226\end{array}$ & $\begin{array}{l}0.08 / 0.92 \\
0.147\end{array}$ & C & $\mathrm{T}$ & $\mathrm{T}$ & $\mathrm{T}$ & $\mathrm{T}$ & $\mathrm{T}$ & C & C & $\mathrm{T}^{\mathrm{a}}$ \\
\hline 8 & rs5963428 & $\mathrm{A} / \mathrm{T}$ & $\begin{array}{l}0.05 / 0.95 \\
0.095\end{array}$ & $\begin{array}{l}0.24 / 0.76 \\
0.365\end{array}$ & $\begin{array}{l}0.43 / 0.47 \\
0.404\end{array}$ & $\mathrm{~T}$ & $\mathrm{~T}$ & $\mathrm{~T}$ & $\mathrm{~T}$ & A & $\mathrm{T}$ & $\mathrm{T}$ & $\mathrm{T}$ & $\mathrm{T}$ \\
\hline 9 & rs12557315 & $\mathrm{C} / \mathrm{T}$ & $\begin{array}{l}0.82 / 0.18 \\
0.295\end{array}$ & $\begin{array}{l}0.80 / 0.20 \\
0.320\end{array}$ & $\begin{array}{l}0.98 / 0.02 \\
0.039\end{array}$ & $\mathrm{C}$ & $\mathrm{T}$ & $\mathrm{C}$ & $\mathrm{C}$ & C & C & $\mathrm{C}$ & $\mathrm{C}$ & $\mathrm{C}$ \\
\hline
\end{tabular}

Abbreviations: JPT, Japanese in Tokyo; CEU, Utah residents with Northern and Western European ancestry from CEPH collection; YRI, Yoruban in Ibadan, Nigeria.

aSurrogated by the nucleotide in this position in Pongo pygmaeus abelii (Orangutan) because it was not available for $P$. troglodytes.

Shaded cells indicate nucleotide bases different from those in $P$. Troglodytes. The segments enclosed by frames indicate those which may have been involved in recombinatory event to form HT4 or generation of HT's 3 and 7 from HT4. 
Association of the three mutant alleles and particular haplotypes The results of the haplotype analysis of the 21 families are summarized in Table 2. Among the nine Japanese probands carrying the p. $\mathrm{R} 40 \mathrm{H}$ mutation, those belonging to families $1-8$, who resided in an area within a radius of $140 \mathrm{~km}$, all had $\mathrm{HT} 1$, whereas family 9, who lived $650 \mathrm{~km}$ away from that area, had HT2. One Spanish family and one Australian family had HT3. In this Australian family, the proband (a girl) bore HT3/8; her mother, 3/7; and father, HT8. Previous analysis had shown that mother carried the mutant allele. Therefore, it was determined that the mutation was linked with the HT3 allele. The Austrian family and one US family shared HT4. The proband in the remaining one US family had HT2. Thus, in the 14 families carrying the p.R40H mutation, this mutation was associated with four different HT's, indicating that it had appeared anew at least four times in the Japanese and the Caucasian populations. Similarly, the p.R277W mutation was associated with three different HT's in our five families carrying this mutation. Three families that carried p.R277W, one Japanese, Korean and US family, shared HT5 each. One of the remaining two US families carried HT6 and the other, HT2. The two Japanese families that carried the p.Y55D mutation shared HT1.

\section{Evolutional order of the haplotypes}

To estimate the evolutional order of these haplotypes, nucleotides in each polymorphic site were compared with those in P. troglodytes (Table 1). HT8 differed only in polymorphic site 7 (T-to-G) from that of P. troglodytes. HT1, HT5, HT6 and HT7 exhibited nucleotide changes in two polymorphic sites, whereas in HT2, HT3 and HT4, there were nucleotide changes in three polymorphic sites.

\section{DISCUSSION}

Although the majority of mutations at the OTC locus are 'private' in patients with neonatal and infantile presentation, ${ }^{11}$ some mutations have been found to recur in unrelated families. ${ }^{12}$ Among mutations associated with late-onset OTCD in male patients, the two mutations, p.R40H and p.R277W, have been most frequently reported in multiple different families. ${ }^{7,12-15,25}$ The p.Y55D mutation has been found only in two unrelated Japanese families. ${ }^{10,16}$ It was not known whether or not the recurring mutations shared a common ancestral origin or had arisen independently. The present study suggests that the p.R40H mutation occurred at least four times, or even five times, if the family 14 is non-Japanese American, in the Japanese and in the Caucasian populations. It appears very likely that those eight Japanese families (1-8) share a common ancestral origin and the mutant allele had been retained in the population in this small area. The other Japanese family (family 9) had a distinct haplotype, however, suggesting this mutation had arisen recurrently. Families 10 and 11 , both Caucasian, may also have a common ancestral origin. Although the Australian family has no known Spanish ancestry, the proband's maternal grandfather (not studied) was of North Italian descent and could conceivably share ancestry with the Spanish family. It remains possible that the mutation occurred recurrently, but it is noteworthy that the allele in these families has a low haplotype frequency (0.033) among

Table 2 Haplotypes of mutant alleles carrying the three mutations

\begin{tabular}{|c|c|c|c|c|c|c|c|c|c|}
\hline Family & $1-6$ & 7 & 8 & 9 & 10 & 11 & 12 & 13 & 14 \\
\hline Mutation & p.R4OH & & & & & & & & \\
\hline Haplotype & 1 & 1 & 1 & 2 & 3 & 3 & 4 & 4 & 2 \\
\hline Reference no. & Harada et al. ${ }^{7 a}$ & Matsuda et al. ${ }^{25}$ & Present report & Present report & Arranz et al. ${ }^{27}$ & Pinner et al. ${ }^{28}$ & Plöchl et al. ${ }^{23}$ & $\begin{array}{l}\text { Tuchman et al., }{ }^{11} \\
\text { Tuchman et al. }{ }^{12}\end{array}$ & \\
\hline Ethnicity & Japanese & Japanese & Japanese & Japanese & Caucasian & Caucasian & Caucasian & Unknown & Unknown \\
\hline $\begin{array}{l}\text { Residential } \\
\text { country }\end{array}$ & Japan & Japan & Japan & Japan & Spain & Australia & Austria & United States & United States \\
\hline \multicolumn{10}{|c|}{ Allele frequency } \\
\hline JPT & 0.408 & & & 0.151 & 0.031 & & 0.133 & & 0.151 \\
\hline CEU & 0.067 & & & 0.155 & 0.033 & & 0.356 & & 0.155 \\
\hline YRI & 0.056 & & & 0.022 & $<0.05^{b}$ & & 0.044 & & 0.022 \\
\hline Family & 15 & 16 & 17 & 18 & 19 & 20 & 21 & & \\
\hline Mutation & p.R277W & & & & & p.Y55D & & & \\
\hline Haplotype & 5 & 5 & 5 & 6 & 2 & 1 & 1 & & \\
\hline Reference no. & Numata et al. ${ }^{10}$ & Kim et al. ${ }^{29}$ & McCullough et al. ${ }^{14}$ & & & Nishiyori et al. ${ }^{16}$ & Numata et al. ${ }^{10}$ & & \\
\hline Ethnicity & Japanese & Korean & Unknown & Unknown & Unknown & Japanese & Japanese & & \\
\hline $\begin{array}{l}\text { Residential } \\
\text { country }\end{array}$ & Japan & Korea & United States & United States & United States & Japan & Japan & & \\
\hline \multicolumn{10}{|c|}{ Allele frequency } \\
\hline JPT & 0.061 & & & $<0.05^{b}$ & 0.151 & 0.408 & & & \\
\hline CEU & 0.175 & & & $<0.05^{b}$ & 0.155 & 0.067 & & & \\
\hline YRI & 0.122 & & & 0.056 & 0.022 & 0.056 & & & \\
\hline
\end{tabular}

Abbreviations: JPT, Japanese in Tokyo; CEU, Utah residents with Northern and Western European ancestry from CEPH collection; YRI, Yoruban in Ibadan, Nigeria.

apatients $1,3,4,8.9$ and 10 in reference Harada et al. ${ }^{7}$

below minimum-allele frequency. 
Caucasians. Families 12 and 13 share a haplotype, but there is insufficient information to say whether this represents the effect of common ancestry or of recurrence on the same haplotype. HT4 is the most common in Caucasians (allele frequency 0.356 in the $\mathrm{CEPH}$ Caucasian samples) and there is no information about the ethnicity of family 13 .

Among the families with the p.R277W mutation, the Japanese and the Korean families (families 15 and 16) could have stemmed from a common ancestor. Family 18 bore HT6, confirming recurrent origin of this mutation.

The families that harbored the p.Y55D mutation (families 20 and 21) shared HT1. This mutation has been found among the Japanese population alone to date. ${ }^{10,16}$ In contrast to the p.R40H and p.R277W mutations, which involve $\mathrm{CpG}$ dinucleotides and hence are likely to recur, this transversion (T-to-G) is not common among single base substitutions in $\mathrm{X}$ chromosome genes in general ${ }^{26}$ and in the OTC gene in particular. ${ }^{12,14,27}$ This characteristic of the p.Y55D mutation would again support that the affected families share a common ancestral origin.

The search for polymorphic markers and the determination of their allelic frequency is required for exploring the origin of an allele. As expected, different types of polymorphic markers in the OTC gene or in its vicinity are known and include SNPs in the coding region, ${ }^{9,17,20}$ in the $5^{\prime}$ untranslated region ${ }^{21}$ and extragenic microsatellite markers. ${ }^{18,19,22}$ Four SNPs, A-to-G in codon 46 in exon 2 (E2 46), A-to-T in intron 3 (IVS3-8nt), A-to-G in intron 4 (IVS4-7nt) and A-to-G in codon 270 of exon 8 (E8 270), were found to be informative among a US population, ${ }^{17}$ though their informativity varied somewhat in the Iberian population and in the Mozambiquan population. ${ }^{18}$ One additional SNP (IVS3-39_insT) was informative in a Spanish population, ${ }^{20}$ indicating that informativity of these SNPs varied between populations. The nine tagged SNPs we used in the present study were all highly informative, except rs5963428, which presented 0.095 heterozygosity in JPT (Table 1). These tagged SNPs were all informative in CEU and YRI also, with the exception of rs12557315 in YRI. We thus considered it appropriate to apply these tagged SNPs to define haplotypes in other ethnicities also. There are several advantages of using these tagged SNPs in determining haplotypes. First, these SNPs generally show high heterozygosity frequency, allowing high informativity. Second, the tagged SNPs are located over the entire span of the OTC gene, reducing misdiagnosis due to an extragenic recombination event when diagnosis by linkage analysis is necessary in the absence of an identifiable mutation. Third, it permits easy differentiation between intragenic recombination and mutation of one particular marker single nucleotide (both rare events). Finally, the analytical efficiency of this technique is high as amplification and data acquisition of up to seven specimens can be completed simultaneously within $90 \mathrm{~min}$ under the conditions employed.

Both the p.R40H and p.R277W mutations arise in CpG dinucleotides, which represent mutational hot spots, consistent with our finding that they have recurred in different populations. Recurrent point mutations occur evenly among most $\mathrm{CpG}$ dinucleotides in the OTC gene. ${ }^{9,12,14,27}$ These two mutations arise in arginine codons. It is not surprising that these single nucleotide changes have a higher chance to recur than others, because four of six codons that encode for arginine contain $\mathrm{CpG}$ dinucleotides. Indeed, scrutiny of a recent OTC mutation update ${ }^{9}$ reveals that 20 discrete mutations have occurred in a total of 15 arginine codons (ratio: $20 / 15=1.33$ ) in the human OTC gene, whereas this ratio is less than 1.0 in the vast majority of codons encoding for other amino acids.
Haplotype 8 differed by one nucleotide from that in P. troglodytes, whereas the other seven HT's exhibited two or three nucleotide differences. This possibly indicates that HT8 is the oldest among the eight HT's identified in the present series. HT's 3 and 4 shared segments consisting of identical nucleotides (polymorphic sites 5-9) and HT's 4 and 7 had jointly another identical segment (polymorphic sites 1-3) (Table 1). It is thus possible that HT3 and HT7 were generated by a recombination of the HT4 allele with another allele, or conversely HT4 was generated by a recombination of HT3 with HT7.

\section{ACKNOWLEDGEMENTS}

We thank Professor Ichiro Matsuda of the Health Sciences University of Hokkaido for his encouraging discussion, Professor Walter Plöchl of the Department of Anesthesiology and Intensive care, Medical University of Vienna and Professor Engelbert Plöchl of the St Johannes Hospital Salzburg, for their cooperation in collecting specimens. This work was supported in part by a grant from the Japan Ministry of Education, Culture, Sport, Science and Technology and by a grant from the Research Center for Innovative Cancer Therapy of the 21th Century COE Program for Medical Science, Kurume University.

1 Lindgren, V., de Martinville, B., Horwich, A. L., Rosenberg, L. E. \& Francke, U. Human ornithine transcarbamylase locus mapped to band Xp21.1 near the Duchenne muscular dystrophy locus. Science 226, 698-700 (1984).

2 Horwich, A. L., Fenton, W. A., Williams, K. R., Kalousek, F., Kraus, J. P., Doolittle, R. F. et al. Structure and expression of a complementary DNA for the nuclear coded precursor of human mitochondrial ornithine transcarbamylase. Science 224, 1068-1074 (1984).

3 Hata, A., Tsuzuki, T., Shimada, K., Takiguchi, M., Mori, M. \& Matsuda, I. Structure of the human ornithine transcarbamylase gene. J. Biochem. 103, 302-308 (1988).

4 Maestri, N. E., Clissold, D. \& Bruislow, S. W. Neonatal onset ornithine transcarbamylase deficiency: a retrospective analysis. J. Pediatr. 134, 268-272 (1999).

5 Finkelstein, J. E., Hauser, E. R., Leonard, C. O. \& Brusilow, S. W. Late-onset ornithine transcarbamylase deficiency in male patients. J. Pediatr. 117, 897-902 (1990).

6 Yoshino, M., Nishiyori, A., Yamashita, F., Abe, H., Tanikawa, K., Ohno, T. et al. Ornithine transcarbamylase deficiency in male adolescence and adulthood. Enzyme 43, 160-168 (1990)

7 Harada, E., Nishiyori, A., Tokunaga, Y., Watanabe, Y., Kuriya, N., Kumashiro, R. et al. Late-onset ornithine transcarbamylase deficiency in male patients: prognostic factors and characteristics of plasma amino acid profile. Pediatr. Int. 48, 105-111 (2006).

8 Tuchman, M., Jaleel, N., Morizono, H., Sheehy, L. \& Lynch, M. G. Mutations and polymorphisms in the human ornithine transcarbamylase gene. Hum. Mutat. 19, 93-107 (2002).

9 Yamaguchi, S., Brailey, L. L., Morizono, H., Bale, A. E. \& Tuchman, M. Mutations and polymorphisms in the human ornithine transcarbamylase (OTC) gene. Hum. Mutat. 27, 626-632 (2006)

10 Numata, S., Harada, E., Maeno, Y., Ueki, I., Watanabe, Y., Fujii, C. et al. Paternal transmission and slow elimination of mutant alleles associated with late-onset ornithine transcarbamylase deficiency in male patients. J. Hum. Genet. 53, 10-17 (2008)

11 Tuchman, M., Morizono, H., Rajagopal, B S., Plante, R J. \& Allenwell, N. M. Identification of 'private' mutations in patients with ornithine transcarbamylase deficiency. J. Inherit. Metab. Dis, 20, 525-527 (1997).

12 Tuchman, M., Plante, R. J., García-Pérez, M. A. \& Rubio, V. Relative frequency of mutations causing ornithine transcarbamylase deficiency in 78 families. Hum. Genet. 97, 274-276 (1996)

13 Matsuda, I. \& Tanase, S. The ornithine transcarbamylase (OTC) gene: mutations in 50 Japanese families with OTC deficiency. Am. J. Med. Genet. 71, 378-383 (1997)

14 McCullough, B. A., Yudkoff, M., Batshaw, M. L., Wilson, J. M., Raper, S. E. \& Tuchman, M. Genotype spectrum of ornithine transcarbamylase deficiency: correlation with the clinical and biochemical phenotype. Am. J. Med. Genet. 93, 313-319 (2000).

15 Nishiyori, A., Yoshino, M., Kato, H., Matsuura, T., Hoshide, R., Matsuda, I. et al. The $\mathrm{R} 4 \mathrm{OH}$ mutation in late onset type of human ornithine transcarbamylase deficiency in male patients. Hum. Genet. 99, 171-176 (1997).

16 Nishiyori, A., Yoshino, M., Tananari, Y., Matsuura, T., Hoshide, R., Matsuda, I. et al. Y55D Mutation in ornithine transcarbamylase associated with late-onset hyperammonemia in a male. Hum. Mutat. 1 (Suppl), 131-133 (1998).

17 Plante, R. J. \& Tuchman, M. Polymorphisms in the human ornithine transcarbamylase gene useful for allele tracking. Hum. Mutat. 12, 289-290 (1998). Mutation in brief \#193 Online.

18 Azevedo, L., Calafell, F., Vilarinho, L. \& Amorim, A. Haplotype analysis and phylogeny of ornithine transcarbamylase polymorphism. Ann. Hum. Genet. 66, 379-385 (2002). 
19 Azevedo, L., Vilarinho, L., Teles, E. L. \& Amorim, A. Ornithine transcarbamylase deficiency: a novel splice site mutation in a family with meiotic recombination and a new useful SNP for diagnosis. Mol. Genet. Metab. 76, 68-70 (2002).

20 Climent, C. \& Rubio, V. H Intragenic polymorphisms and haplotype analysis in the ornithine transcarbamylase (OTC) gene and their relevance for tracking the inheritance of OTC deficiency. Hum. Mutat. 20, 407-408 (2002). Mutation in Brief \#547 Online.

21 Azevedo, L., Stolnaja, L., Tietzeova, E., Hrebicek, M., Hruba, E., Vilarinho, L. et al. New polymorphic sites within ornithine transcarbamylase gene: population genetics studies and implications for diagnosis. Mol. Genet. Metab. 78, 152-157 (2003).

22 Azevedo, L., Climent, C., Vilarinho, L., Calafell, F. \& Amorim, A. Evidence for mutational cis-acting factors affecting mutagenesis in the ornithine transcarbamylase gene. Hum. Mutat. 24, 273 (2004). Mutation in Brief \#739 Online.

23 Plöchl, W. Spiss, C. K. \& Plöchl, E. Death after transplantation of a liver from a donor with unrecognized ornithine transcarbamylase deficiency. N. Eng. J. Med. 341, 921-922 (1999).

24 Barrett, J. C., Fry, B., Maller, J. \& Daly, M. J. Haploview: analysis and visualization of LD and haplotype maps. Bioinformatics 21, 263-265 (2005) [PubMed ID 15297300].

25 Matsuda, I., Matsuura, T., Nishiyori, A., Komaki, S., Hoshide, R., Matsumoto, T. et al. Phenotypic variability in male patients carrying the mutant ornithine transcarbamylase (OTC) allele Arg4OHis, ranging from a child with an unfavourable prognosis to an asymptomatic order adult. J. Med. Genet. 33, 645-648 (1996).

26 Antonarakis, S. E., Krawczak, M. \& Cooper, D. N. The nature and mechanism of human gene mutation. In The Metabolic and Molecular Bases of Inherited Disease (eds. Scriver, C.R., Beaudet, A.L., Sly, W.S., Valle, D.) 8th edn. 343-377 (McGraw-Hill, New York, 2001).

27 Arranz, J. A., Riudor, E., Marco-Marín, C. \& Rubio, V. Estimation of the total number of diseases-causing mutations in ornithine transcarbamylase (OTC) deficiency. Value of the OTC structure in predicting a mutation pathogenic potential. J. Inherit. Metab. Dis. 30, 217-226 (2007).

28 Pinner, J. R., Freckmann, M.- L., Yoshino, M. \& Kirk, E. P. Female heterozygotes for the hypomorphic $\mathrm{R} 4 \mathrm{OH}$ mutation can have ornithine transcarbamylase deficiency and present in early adolescence: two cases. J. Med. Case Report, submitted.

29 Kim, G.- H., Choi, J.- H., Lee, H.- H., Park, S., Kim, S.- S. \& Yoo, H.- W. Identification of novel mutations in the human ornithine transcarbamylase (OTC) gene of Korean patients with OTC deficiency and transient expression of the mutant proteins in vitro. Hum. Mutat. 27, 1159 (2006). Mutation in Brief \#936 Online. 